\title{
Maximum principles and symmetry results for a class of fully nonlinear elliptic PDEs
}

\author{
Cristian Enache
}

\begin{abstract}
This paper is concerned with a class of boundary value problems for fully nonlinear elliptic PDEs involving the $p$-Hessian operator. We first derive a maximum principle for a suitable function involving the solution $u(\mathbf{x})$ and its gradient. This maximum principle is then applied to obtain some sharp estimates for the solution and the magnitude of its gradient. We also investigate some symmetry properties of $\Omega$ or $u(\mathbf{x})$ under specific boundary condition or geometry of $\Omega$.
\end{abstract}

Mathematics Subject Classification (2000). Primary 35B50, 35R35;

Secondary 35B45.

Keywords. Fully nonlinear elliptic equations, Maximum principles, A priori bounds, Overdetermined problems, Radial solutions.

\section{Introduction}

Let $\Omega \subset \mathbb{R}^{N}, N \geq 2$, be a bounded domain containing the origin and let $p \in\{1, \ldots, N\}$. This paper is devoted to the study of the following type of boundary value problems

$$
\begin{cases}S_{p}\left(D^{2} u\right)=g(u) h\left(|\nabla u|^{2}\right) & \text { in } \Omega \\ u=0 & \text { on } \partial \Omega\end{cases}
$$

where $g$ and $h$ are positive $C^{1}$ functions assumed to satisfy the following condition

$$
h^{-1 / p} \frac{g^{\prime}}{g}+2\left(\begin{array}{c}
N \\
p
\end{array}\right)^{-1 / p} g^{1 / p} \frac{h^{\prime}}{h} \geq 0,
$$


$D^{2} u$ is the Hessian matrix of $u$ and $S_{p}\left(D^{2} u\right)$ is the $p$ th elementary symmetric function of the eigenvalues $\lambda_{1}, \ldots, \lambda_{N}$ of $D^{2} u$, i.e.

$$
S_{p}\left(D^{2} u\right) \equiv S_{p}\left(\lambda_{1}, \ldots, \lambda_{N}\right):=\sum_{1 \leq i_{1}<\cdots<i_{p} \leq N} \lambda_{i_{1}} \cdots \lambda_{i_{p}} .
$$

We note that the $p$-Hessian operator $S_{p}\left(D^{2} u\right)$ is homogeneous of degree $p$ and, for $p>1$, it is elliptic only if it is restricted to the class of $p$-convex functions

$$
\Gamma_{p}(\Omega)=\left\{u \in C^{2}(\Omega): S_{i}\left(D^{2} u\right) \geq 0 \text { in } \Omega, i=1, \ldots, p\right\} .
$$

Therefore, Eq. (1.1) is called elliptic for a particular solution $u(\mathbf{x})$ if the matrix

$$
A^{*}:=\left(S_{p}^{i j}\right), \quad \text { with } S_{p}^{i j}:=\partial S_{p} / \partial u_{, i j},
$$

is positive definite.

Our results will apply only on the classical solutions of Eq. (1.1) for which the ellipticity condition is satisfied. We refer to such solutions as admissible solutions of Eq. (1.1). The existence of negative admissible solutions for the boundary value problems of type (1.1) is known and has been well studied by Trudinger in [13]. Particular cases of our work have been considered and investigated in some previous works, such as, e.g. Ma [4] (when $p=N=2$ and $g h=$ const.) and Philippin and Safoui $[8,9]$ (when $p=N$ and $g h=$ const.) for maximum principles or Payne and Schaefer [7] and Brandolini and co. [2] (when $g h=$ const.) for symmetry results. In order to handle our more general problems, we make use of the techniques developed in these papers. The main tools of our investigations will be Hopf's first and second maximum principles [10] and some geometric arguments involving the curvatures of the level sets of $u$.

The outline of the paper is as follows. In Sect. 2 we prove a maximum principle for an appropriate functional combination of the solution and its gradient, which generalizes the maximum principles obtained by Ma [4] and Philippin and Safoui [8,9]. Some possible applications of our maximum principle will be given in Sect. 3, where we derive various sharp bounds for $u(\mathbf{x})$ and $|\nabla u|^{2}$, i.e. bounds that cannot be improved without using additional informations. Finally, in Sect. 4 we investigate some symmetry properties of $\Omega$ and $u$ under specific boundary conditions or geometry of $\Omega$.

For convenience, notice that throughout the paper the comma is used to indicate differentiation and the summation from 1 to $N$ is understood on repeated indices. Moreover, when appropriate we use the notations $u_{\min }:=$ $\min _{\bar{\Omega}} u(\mathbf{x})$ and $q_{0}:=\max _{\partial \Omega}|\nabla u|$.

\section{A maximum principle}

For convenience, we first introduce some notations. Let $u(\mathbf{x})$ be a $C^{2}$ function and let $k_{1}(t), \ldots, k_{N-1}(t)$ be the principal curvatures of the level set 
$L_{t}:=\{u=t\}$, where $t$ is a regular value of $u$. For $q \in\{1, \ldots, N-1\}$, we define the $q$-curvature of $L_{t}$ by

$$
H_{q}\left(L_{t}\right):=S_{q}\left(k_{1}(t), \ldots, k_{N-1}(t)\right),
$$

where $S_{q}$ denotes again the $q$ th elementary symmetric function (of $N-1$ variables in this case!). We also set $H_{0} \equiv 1, H_{N} \equiv 0$ and we note that $H_{1}=$ $(N-1) K$ and $H_{N-1}=G$, where $K$ denotes the mean curvature of $L_{t}$ and $G$ denotes the Gaussian curvature of $L_{t}$. Moreover, we recall that any level set of a $p$-convex function has to be a $(p-1)$-convex set (see Trudinger [13]), that is a set whose principal curvatures $k_{1}, \ldots, k_{N-1}$ satisfy

$$
H_{i}=S_{i}\left(k_{1}, \ldots, k_{N-1}\right) \geq 0, \text { for } i=1, \ldots, p-1 .
$$

We now state the following maximum principle:

Theorem 2.1. Assume that $u(\mathbf{x}) \in C^{3}(\Omega) \cap C^{2}(\bar{\Omega})$ is an admissible solution of Eq. (1.1), with convex level sets. Let $\Phi(\mathbf{x})$ be an auxiliary function defined by

$$
\Phi(\mathbf{x}):=\int_{0}^{|\nabla u|^{2}} h^{-1 / p}(s) d s-2\left(\begin{array}{c}
N \\
p
\end{array}\right)^{-1 / p} \int_{0}^{u} g^{1 / p}(s) d s .
$$

Then the function $\Phi(\mathbf{x})$ takes its maximum value on the boundary $\partial \Omega$.

Proof. Differentiating (2.3), we obtain successively

$$
\begin{aligned}
\Phi_{, k}= & 2 h^{-1 / p} u_{, k j} u_{, j}-2\left(\begin{array}{c}
N \\
p
\end{array}\right)^{-1 / p} g^{1 / p} u_{, k}, \\
S_{p}^{k l} \Phi_{, k l}= & -\frac{4}{p} h^{-(1 / p)-1} h^{\prime} S_{p}^{k l} u_{, k j} u_{, j} u_{, l i} u_{, i} \\
& +2 h^{-1 / p}\left[S_{p}^{k l} u_{, k l j} u_{, j}+S_{p}^{k l} u_{, k j} u_{, j l}\right] \\
& -\frac{2}{p}\left(\begin{array}{c}
N \\
p
\end{array}\right)^{-1 / p} g^{(1 / p)-1} g^{\prime} S_{p}^{k l} u_{, k} u_{, l}-2\left(\begin{array}{c}
N \\
p
\end{array}\right)^{-1 / p} g^{1 / p} S_{p}^{k l} u_{, k l},
\end{aligned}
$$

where $\left(S_{p}^{k l}\right)$ is the positive definite matrix defined in (1.5).

We will compute separately each term of (2.5). First, we note that Euler identity for homogenous functions and (1.1) give

$$
S_{p}^{k l} u_{, k l}=p S_{p}\left(D^{2} u\right)=p g(u) h\left(|\nabla u|^{2}\right) .
$$

Next, we will make use of the fact that on every level set $L_{t}=\{u=t\}$ the following identity holds (see Brandolini and co. [2]):

$$
S_{p}^{i j} u_{, i} u_{, l} u_{, l j}=S_{p}|\nabla u|^{2}-H_{p}|\nabla u|^{p+1} .
$$

Therefore, making use of (2.4) and (2.7), we can write other two terms of (2.5) in the following form

$$
\begin{aligned}
S_{p}^{k l} u_{, k j} u_{, j} u_{, l i} u_{, i} & =\left(\begin{array}{c}
N \\
p
\end{array}\right)^{-1 / p} h^{1 / p} g^{1 / p}\left[S_{p}|\nabla u|^{2}-H_{p}|\nabla u|^{p+1}\right]+\cdots, \\
S_{p}^{k l} u_{, k} u_{, l} & =\left(\begin{array}{c}
N \\
p
\end{array}\right)^{1 / p} h^{-1 / p} g^{-1 / p}\left[S_{p}|\nabla u|^{2}-H_{p}|\nabla u|^{p+1}\right]+\cdots,
\end{aligned}
$$

in $\Omega \backslash\{\mathbf{K}\}$, where the dots stand for terms containing $\Phi_{, k}$ and $\mathbf{K} \in \Omega$ is the unique critical point of $u$ (since $u$ has convex level sets). 
In order to compute $S_{p}^{k l} u_{, k l j} u_{, j}$, we combine the definition of $S_{p}^{k l}$, and (2.4) to obtain

$$
S_{p}^{k l} u_{, k l j} u_{, j}=\left[g^{\prime} h|\nabla u|^{2}+2\left(\begin{array}{c}
N \\
p
\end{array}\right)^{-1 / p} g^{(1 / p)+1} h^{1 / p} h^{\prime}|\nabla u|^{2}\right]+\cdots,
$$

where the dots have the same meaning as above.

Finally, in order to compute $S_{p}^{k l} u_{, k j} u_{, j l}$, we first notice that

$$
S_{p}^{k l} u_{, k j} u_{, j l}=S_{p} S_{1}-(p+1) S_{p+1},
$$

and then we make use of the classical Newton inequality for means of $p$-convex functions (see Hardy, Littlewood and Pólya [3]):

$$
\left(\frac{S_{r}}{\left(\begin{array}{c}
N \\
r
\end{array}\right)}\right)^{1 / r} \geq\left(\frac{S_{s}}{\left(\begin{array}{c}
N \\
s
\end{array}\right)}\right)^{1 / s} \quad \text { if } 1 \leq r<s \leq p+1,
$$

with equality if and only if $\lambda_{1}=\cdots=\lambda_{N}$. In particular, (2.12) gives

$$
S_{1} \geq N\left(\frac{S_{p}}{\left(\begin{array}{c}
N \\
p
\end{array}\right)}\right)^{1 / p}, S_{p+1} \leq\left(\begin{array}{c}
N \\
p+1
\end{array}\right)\left(\frac{S_{p}}{\left(\begin{array}{c}
N \\
p
\end{array}\right)}\right)^{1+1 / p} .
$$

Replacing (2.13) into (2.11), we obtain

$$
S_{p}^{k l} u_{, k j} u_{, j l} \geq p\left(\begin{array}{c}
N \\
p
\end{array}\right)^{-1 / p} g^{1+1 / p}(u) h^{1+1 / p}\left(|\nabla u|^{2}\right) .
$$

Inserting now $(2.6),(2.8),(2.9),(2.10)$ and (2.14) in (2.5) we obtain

$$
\begin{aligned}
S_{p}^{k l} \Phi_{, k l}+W_{k} \Phi_{, k} \geq & 2|\nabla u|^{2}\left[g h\left(1-\frac{1}{p}\right)+\frac{1}{p} H_{p}|\nabla u|^{p-1}\right] \\
& \cdot\left[2\left(\begin{array}{c}
N \\
p
\end{array}\right)^{-1 / p} g^{1 / p} \frac{h^{\prime}}{h}+h^{-1 / p} \frac{g^{\prime}}{g}\right] \text { in } \Omega \backslash\{\mathbf{K}\},
\end{aligned}
$$

where $W_{k}$ is the $k$ th component of a vector field regular throughout $\Omega$. Since $u$ has convex level sets, the principal curvatures are defined in all points of the level sets of $u$ and they are nonnegative. Consequently, $H_{p}$ is nonnegative in (2.15). It then follows from (1.2) and Hopf's first maximum principle [10] that $\Phi(\mathbf{x})$ takes its maximum value either on $\partial \Omega$ or at $\mathbf{K} \in \Omega$ (which is excluded if we evaluate (2.5) at $\mathbf{K}$, too) and the proof is achieved.

We remark that the auxiliary function $\Phi(\mathbf{x})$ defined in (2.3) becomes constant when $g$ and $h$ are constant functions. Therefore, for $g \neq$ const. or $h \neq$ const., the maximum principle obtained in Theorem 2.1 is not the best possible. We also notice that, if for $p=1$ and $p=N$ it is known that (1.1) has solutions with convex level sets when $\Omega$ is convex, for $p \in\{2, \ldots, N-1\}$ this property remains unknown (such a convexity result was recently obtained by Ma and $\mathrm{Xu}[5]$ only for the case $N=3, p=2, f=g \equiv 1)$. However, in Sect. 4 it will be shown that the level sets of $u(\mathbf{x})$ are balls when $\Omega$ is a ball. 


\section{A priori bounds}

In this section we apply the maximum principle of the previous section to two fully nonlinear boundary value problems of type (1.1). We would expect the maximum principle to yield inequalities for the solution and its gradient.

Problem 3.1. Assume that $u(\mathbf{x}) \in C^{3}(\Omega) \cap C^{2}(\bar{\Omega})$ is an admissible solution, with convex level sets, of the following boundary value problem:

$$
\begin{cases}S_{p}\left(D^{2} u\right)=g(u) & \text { in } \Omega \\ u=0 & \text { on } \partial \Omega\end{cases}
$$

where $g$ is a positive $C^{1}$ function, with $g^{\prime} \geq 0$. Since the cases $p=1$ and $p=N$ have already been treated by Payne and Philippin [6], respectively Philippin and Safoui [8], we consider here only the case $p \in\{2, \ldots, N-1\}$.

With $h \equiv 1$ in (1.1), the auxiliary function $\Phi(\mathbf{x})$ defined in (2.3) becomes

$$
\Phi(\mathbf{x})=|\nabla u|^{2}-2\left(\begin{array}{c}
N \\
p
\end{array}\right)^{-1 / p} \int_{0}^{u} g^{1 / p}(s) d s .
$$

Theorem 2.1 implies that $\Phi(\mathbf{x})$ takes its maximum value at some point $\mathbf{P} \in \partial \Omega$, or

$$
-2\left(\begin{array}{c}
N \\
p
\end{array}\right)^{-1 / p} \int_{0}^{u} g^{1 / p}(s) d s \leq q_{0}^{2}-|\nabla u|^{2} \text { in } \Omega .
$$

Evaluating (3.3) at the critical point of $u$, we obtain the following inequality:

$$
q_{0}^{2} \geq-2\left(\begin{array}{c}
N \\
p
\end{array}\right)^{-1 / p} \int_{0}^{u_{\min }} g^{1 / p}(s) d s .
$$

Next, we construct an upper bound for $q_{0}^{2}$ in terms of the geometry of $\Omega$. First, we notice that $\Phi(\mathbf{x})$ and $|\nabla u|^{2}$ take their maximum value at the same point $\mathbf{P} \in \partial \Omega$. It then follows that, in normal coordinates with respect to the boundary, the hessian matrix of $u$ takes the following form at $\mathbf{P}$ (see Lemma 8 from [9]):

$$
D^{2} u(\mathbf{P})=\operatorname{diag}\left(k_{1} u_{, n}, \ldots, k_{N-1} u_{, n}, u_{, n n}\right),
$$

where $k_{1}, \ldots, k_{N-1}$ are the principal curvatures of $\partial \Omega$ and $u_{, n}, u_{, n n}$ are the first and second outward normal derivatives of $u$ at $\mathbf{P} \in \partial \Omega$.

Now, the differential equation (3.1) evaluated on $\partial \Omega \in C^{2}$ gives

$$
S_{p}\left(\lambda_{1}, \ldots, \lambda_{N}\right) \equiv S_{p}\left(\lambda_{1}, \ldots, \lambda_{N-1}\right)+\lambda_{N} S_{p-1}\left(\lambda_{1}, \ldots, \lambda_{N-1}\right)=g(0) .
$$

Making use of (3.5), we may rewrite (3.6) at the point $\mathbf{P}$ as

$$
|\nabla u|^{p} H_{p}\left(k_{1}, \ldots, k_{N-1}\right)+|\nabla u|^{p-1} u_{, n n} H_{p-1}\left(k_{1}, \ldots, k_{N-1}\right)=g(0) .
$$

On the other hand, since $\Phi(\mathbf{x})$ takes its maximum value at the point $\mathbf{P}$, we have $\partial \Phi / \partial n(\mathbf{P}) \geq 0$, or

$$
u_{, n n} \geq\left(\begin{array}{c}
N \\
p
\end{array}\right)^{-1 / p} g^{1 / p}(0) \text { at } \mathbf{P} .
$$

The insertion of (3.8) into (3.7) leads us to

$$
g(0) \geq|\nabla u|^{p} H_{p}+|\nabla u|^{p-1}\left(\begin{array}{c}
N \\
p
\end{array}\right)^{-1 / p} g^{1 / p}(0) H_{p-1} .
$$


Moreover, if we make use of the arithmetic-geometric inequality in (3.9), we obtain

$$
|\nabla u|^{2 p-1} \leq \frac{1}{4}\left(\begin{array}{c}
N \\
p
\end{array}\right)^{1 / p} \frac{g^{2-(1 / p)}(0)}{4 H_{p} H_{p-1}} \leq \frac{1}{4}\left(\begin{array}{c}
N \\
p
\end{array}\right)^{1 / p} g^{2-(1 / p)}(0) H^{-1},
$$

where $H:=\min _{\partial \Omega}\left\{H_{p} H_{p-1}\right\}$. For an appropriate form of $g$, combining (3.10) and (3.4) a lower bound may be obtained for $u_{\text {min }}$.

Problem 3.2. Assume that $u(\mathbf{x}) \in C^{3}(\Omega) \cap C^{2}(\bar{\Omega})$ is an admissible solution, with convex level sets, of the following boundary value problem:

$$
\begin{cases}S_{p}\left(D^{2} u\right)=h\left(|\nabla u|^{2}\right) & \text { in } \Omega \\ u=0 & \text { on } \partial \Omega\end{cases}
$$

where $h$ is a positive $C^{1}$ function, with $h^{\prime} \geq 0$. We consider again only the case $p \in\{2, \ldots, N-1\}$, since the cases $p=1$ and $p=N$ were already treated in [6] and [8].

With $g \equiv 1$ in (1.1), the auxiliary function $\Phi(\mathbf{x})$ defined in (2.3) becomes

$$
\Phi(\mathbf{x})=\int_{0}^{|\nabla u|^{2}} h^{-1 / p}(s) d s-2\left(\begin{array}{c}
N \\
p
\end{array}\right)^{-1 / p} u .
$$

Then, Theorem 2.1 implies that $\Phi(\mathbf{x})$ takes its maximum value at some point $\mathbf{Q} \in \partial \Omega$, or

$$
-2\left(\begin{array}{l}
N \\
p
\end{array}\right)^{-1 / p} u \leq \int_{0}^{q_{0}^{2}} h^{-1 / p}(s) d s-\int_{0}^{|\nabla u|^{2}} h^{-1 / p}(s) d s .
$$

Evaluating (3.13) at the critical point of $u$, we obtain

$$
-2\left(\begin{array}{c}
N \\
p
\end{array}\right)^{-1 / p} u_{\min } \leq \int_{0}^{q_{0}^{2}} h^{-1 / p}(s) d s .
$$

Next, we will construct an upper bound for $q_{0}$. First, since $\Phi(\mathbf{x})$ and $|\nabla u|^{2}$ take their maximum value at the same point $\mathbf{Q} \in \partial \Omega$, the differential equation (3.11) evaluated at $\mathbf{Q}$ gives

$$
|\nabla u|^{p} H_{p}+|\nabla u|^{p-1} u_{, n n} H_{p-1}=h\left(q_{0}^{2}\right) .
$$

On the other hand, $\partial \Phi / \partial n(\mathbf{Q}) \geq 0$, so

$$
u_{, n n}(\mathbf{Q}) \geq\left(\begin{array}{c}
N \\
p
\end{array}\right)^{-1 / p} h^{1 / p}\left(q_{0}\right)
$$

Inserting (3.16) into (3.15), we have

$$
h\left(q_{0}^{2}\right) \geq|\nabla u|^{p} H_{p}+|\nabla u|^{p-1}\left(\begin{array}{c}
N \\
p
\end{array}\right)^{-1 / p} h^{1 / p}\left(q_{0}^{2}\right) H_{p-1} .
$$

Moreover, using the arithmetic-geometric inequality in (3.17), we obtain

$$
|\nabla u|^{2 p-1} \leq \frac{1}{4}\left(\begin{array}{c}
N \\
p
\end{array}\right)^{1 / p} \frac{h^{2-(1 / p)}\left(q_{0}^{2}\right)}{H_{p} H_{p-1}} \leq \frac{1}{4}\left(\begin{array}{c}
N \\
p
\end{array}\right)^{1 / p} h^{2-(1 / p)}\left(q_{0}^{2}\right) H^{-1} .
$$

For an appropriate form of $h$, combining (3.18) and (3.14), a lower bound for $u_{\text {min }}$ may be obtained. 


\section{Some symmetry results}

In this section, we first investigate the case when $\Omega$ is a ball in (1.1) and show that, under some specific conditions on the data, the solution must be radial. In the second part of this section, we consider two classes of nonstandard overdetermined problems for some fully nonlinear equations of type (1.1). In each case it will be shown that if a solution exists, then $\Omega$ must be the interior of a ball.

Theorem 3.1. Assume that $\Omega$ is an $N$-dimensional ball, i.e. $\Omega=B(O, R)$. Let $u \in C^{3}(\Omega) \cap C^{1}(\bar{\Omega})$ be an admissible solution of $(1.1)$, where $g$ and $h$ are positive $C^{1}$ functions, with $g^{\prime} \geq 0$. Then $u(\mathbf{x})$ is radially symmetric, i.e. we have $u(\mathbf{x})=u(|\mathbf{x}|)$.

Proof. For the proof, we take up an idea of Aviles [1] (see also Philippin and Safoui [8]) and adapt it to our context. Basically, the idea of the proof consists of showing that

$$
x_{k} u_{, l}-x_{l} u_{, k} \equiv 0 \text { in } \Omega, \text { for all } k \neq l .
$$

To this end, let us consider the function $w^{k l}(\mathbf{x})$ defined by

$$
w^{k l}(\mathbf{x}):=x_{k} u_{, l}-x_{l} u_{, k}
$$

We compute successively

$$
\begin{aligned}
w_{, i}^{k l} & =\delta_{i k} u_{, l}+x_{k} u_{, i l}-\delta_{i l} u_{, k}-x_{l} u_{, i k}, \\
w_{, i j}^{k l} & =\delta_{i k} u_{, l j}+\delta_{j k} u_{, i l}+x_{k} u_{, i j l}-\delta_{i l} u_{, j k}-\delta_{j l} u_{, i k}-x_{l} u_{, i j k},
\end{aligned}
$$

where $\delta_{k l}$ is the Kronecker symbol $\left(\delta_{k l}=1\right.$ if $k=l$ and $\delta_{k l}=0$ if $\left.k \neq l\right)$. Multiplying (4.4) by $S_{p}^{i j}$, we obtain

$$
S_{p}^{i j} w_{, i j}^{k l}=S_{p}^{k j} u_{, j l}-S_{p}^{l j} u_{, j k}+S_{p}^{k i} u_{, i l}-S_{p}^{i l} u_{, i k}+x_{k} S_{p}^{i j} u_{, i j l}-x_{l} S_{p}^{i j} u_{, i j k}
$$

Next, since $A:=\left(D^{2} u\right)$ and $A^{*}:=\left(S_{p}^{i j}\right)$ are symmetric matrices which commute, i.e. $A^{*} A=A A^{*}$, then $A^{*} A$ is a symmetric matrix, too. Therefore, (4.5) leads us to

$$
S_{p}^{i j} w_{, i j}^{k l}-2 g \frac{\partial h}{\partial\left(|\nabla u|^{2}\right)} u_{, i} w_{, i}^{k l}-\frac{\partial g}{\partial u} h w^{k l}=0 .
$$

Now, since the matrix $A^{*}:=\left(S_{p}^{i j}\right)$ is positive definite, Eq. (4.6) is uniformly elliptic in $\Omega$. Moreover, from the hypothesis of the theorem we have $w^{k l}=0$ on $\partial \Omega$. Therefore, with $g^{\prime}, h \geq 0$ in (4.6), the classical uniqueness result (see Protter and Weinberger [10]) implies that

$$
w^{k l} \equiv 0 \text { in } \Omega \text {, for all } k \neq l,
$$

and the thesis follows.

Let $h(\mathbf{x})$ be the so-called support function or Minkowski function on $\partial \Omega$, i.e.

$$
h(\mathbf{x}):=\mathbf{x} \cdot \mathbf{n}=x_{i} \frac{u_{, i}}{|\nabla u|} \text { for all } \mathbf{x} \in \partial \Omega
$$


where $\mathbf{x}$ is the radius vector from the origin inside $\Omega$ and $\mathbf{n}$ is the normal vector directed outward $\partial \Omega$.

In a seminal paper in 1971, Serrin [12] showed that the only bounded domain $\Omega \subset \mathbb{R}^{N}(N \geq 2)$, with $\partial \Omega \in C^{2}$, on which the solution to the $N$ dimensional torsion problem $(\Delta u=1$ in $\Omega, u=0$ on $\partial \Omega)$ has a constant outward normal derivative on $\partial \Omega$, is the $N$-dimensional ball. In 1994, Payne and Schaefer [7] showed that if the solution of the $N$-dimensional torsion problem has its outward normal derivative equal to the support function on $\partial \Omega$, then $\Omega$ must be the interior of a ball. In what follows, we extend these results to the case of $p$-Hessian equations:

Theorem 3.2. Assume that $\Omega$ is strictly convex. Let $u(\mathbf{x}) \in C^{2}(\bar{\Omega})$ be an admissible solution of the following boundary value problem:

$$
\left\{\begin{array}{ll}
S_{p}\left(D^{2} u\right)=\left(\begin{array}{l}
N \\
p
\end{array}\right) & \text { in } \Omega \\
u=0 & \text { on } \partial \Omega
\end{array}, p \in\{2, \ldots, N\} .\right.
$$

If $u$ also satisfies one of the following further boundary conditions

(i)

$$
H_{p-1}|\nabla u|^{p+1}=\left(\begin{array}{c}
N-1 \\
p-1
\end{array}\right) \text { on } \partial \Omega
$$

(ii)

$$
|\nabla u|=h \text { on } \partial \Omega
$$

then $\Omega$ must be a ball.

Proof. (i) First, we notice that a direct computation shows that for all $j \in\{1, \ldots, N\}$, the vector field $\left(S_{p}^{1 j}\left(D^{2} u\right), \ldots, S_{p}^{N j}\left(D^{2} u\right)\right)$ is divergent free. Therefore, (2.6) implies that $S_{p}\left(D^{2} u\right)$ may be written in the following divergence form:

$$
S_{p}\left(D^{2} u\right)=\frac{1}{p}\left(S_{p}^{i j}\left(D^{2} u\right) u_{, j}\right)_{, i} .
$$

Next, we remind the following pointwise identity (see Reilly [11] or Trudinger $[14])$

$$
S_{p}^{i j}\left(D^{2} u\right) u_{, i} u_{, j}=H_{p-1}|\nabla u|^{p+1} \text { for all } p \in\{1, \ldots, N\} .
$$

From Newton inequality (2.12) and (4.9), we have

$$
\frac{\Delta u}{N} \geq \frac{S_{p}\left(D^{2} u\right)}{\left(\begin{array}{c}
N \\
p
\end{array}\right)} .
$$

On the other hand, making use of Green formula, (4.12), (4.13) and (4.10), we obtain

$$
\int_{\Omega}\left(\frac{\Delta u}{N}-\frac{S_{p}\left(D^{2} u\right)}{\left(\begin{array}{l}
N \\
p
\end{array}\right)}\right) d x=0 .
$$

This implies that we have equality in (4.14). Consequently, the eigenvalues of the hessian matrix $D^{2} u$ are all equal at every point of $\Omega$. This fact and 
Eq. (4.9) imply that $D^{2} u$ is the identity matrix and the result follows in this case.

(ii) Using successively the Newton inequality, Green formula and the overdetermined condition (4.11), we obtain

$$
|\Omega|=\int_{\Omega} \frac{S_{p}\left(D^{2} u\right)}{\left(\begin{array}{l}
N \\
p
\end{array}\right)} \leq \int_{\partial \Omega} \frac{\Delta u}{N}=\frac{1}{N} \int_{\partial \Omega}|\nabla u|=\frac{1}{N} \int_{\partial \Omega} h=|\Omega| .
$$

This implies that $\Delta u=N$, from which the result follows.

\section{References}

[1] Aviles, P.: Symmetry theorems related to Pompeiu's problem. Amer. J. Math. 108, 1023-1036 (1986)

[2] Brandolini, B., Nitsch, C., Salani, P., Trombetti, C.: Serrin type overdetermined problems: an alternative proof. Arch. Ration. Mech. Anal. 190, 267-280 (2008)

[3] Hardy, G.H., Littlewood, J.E., Pólya, G.: Inequalities. Cambridge Mathematical Library. Cambridge University Press, Cambridge (1998)

[4] Ma, X.-N.: A necessary condition of solvability for the capillarity boundary of Monge-Ampère equations in two dimensions. Proc. Am. Math. Soc. 127, 763769 (1999)

[5] Ma, X.-N., Xu, L.: The convexity of solution of a class Hessian equation in bounded convex domain in $R^{3}$. J. Funct. Anal. 255, 1713-1723 (2008)

[6] Payne, L.E., Philippin, G.A.: Some maximum principles for nonlinear elliptic equations in divergence form with applications to capillary surfaces and to surfaces of constant mean curvature. Nonlinear Anal. 3, 193-211 (1979)

[7] Payne, L.E., Schaefer, P.W.: On overdetermined boundary value problems for the Biharmonic Operator. J. Math. Anal. Appl. 187, 598-616 (1994)

[8] Philippin, G.A., Safoui, A.: Some maximum principle and symmetry results for a class of boundary value problems involving Monge-Ampère equation. Math. Models Methods Appl. Sci. 11, 1073-1080 (2001)

[9] Philippin, G.A., Safoui, A.: Some applications of the maximum principle to a variety of fully nonlinear elliptic PDE's. Z. Angew. Math. Phys. 54, 739-755 (2003)

[10] Protter, M.H., Weinberger, H.F.: Maximum principles in differential equations. Springer, Berlin (1975)

[11] Reilly, R.C.: On the Hessian of a function and the curvatures of its graph. Michigan Math. J. 20, 373-383 (1973)

[12] Serrin, J.: A symmetry problem in potential theory. Arch. Rational Mech. Anal. 43, 304-318 (1971) 
[13] Trudinger, N.S.: On the Dirichlet problem for Hessian equations. Acta Math. 175, 151-164 (1995)

[14] Trudinger, N.S.: On new isoperimetric inequalities and symmetrization. J. Reine Angew. Math. 488, 203-220 (1997)

C. Enache

Department of Mathematics and Informatics, Ovidius University,

900527 Constanta,

Romania

e-mail: cenache@univ-ovidius.ro

Received: 17 May 2009.

Accepted: 12 March 2010. 